\title{
Compressive deformation of ultralong amyloid fibrils
}

\author{
Raffaella Paparcone · Steven Cranford • \\ Markus J. Buehler
}

Received: 12 July 2010 / Revised: 12 October 2010 / Accepted: 12 October 2010

(C) The Chinese Society of Theoretical and Applied Mechanics and Springer-Verlag GmbH 2010

\begin{abstract}
Involved in various neurodegenerative diseases, amyloid fibrils and plaques feature a hierarchical structure, ranging from the atomistic to the micrometer scale. At the atomistic level, a dense and organized hydrogen bond network is resembled in a beta-sheet rich secondary structure, which drives a remarkable stiffness in the range of 10-20 GPa, larger than many other biological nanofibrils, a result confirmed by both experiment and theory. However, the understanding of how these exceptional mechanical properties transfer from the atomistic to the nanoscale remains unknown. Here we report a multiscale analysis that, from the atomistic-level structure of a single fibril, extends to the mesoscale level, reaching size scales of hundreds of nanometers. We use parameters directly derived from full atomistic simulations of $\mathrm{A} \beta(1-40)$ amyloid fibrils to parameterize a mesoscopic coarse-grained model, which is used to reproduce the elastic properties of amyloid fibrils. We then apply our mesoscopic model in an analysis of the buckling behavior of amyloid fibrils with different lengths and report
\end{abstract}

The project was supported by the Office of Naval Research (NN00014-08-1-0844) and NSF-MRSEC (DMR-0819762).

R. Paparcone · S. Cranford · M. J. Buehler $(\varangle)$

Laboratory for Atomistic and Molecular Mechanics,

Department of Civil and Environmental Engineering,

Massachusetts Institute of Technology,

77 Massachusetts Ave. Room 1-235 A\& B, Cambridge, MA, USA

e-mail: mbuehler@MIT.EDU

S. Cranford · M. J. Buehler

Center for Materials Science and Engineering,

Massachusetts Institute of Technology, 77 Massachusetts Ave., Cambridge, MA, USA

\section{J. Buehler}

Center for Computational Engineering,

Massachusetts Institute of Technology, 77 Massachusetts Ave.,

Cambridge, MA, USA a comparison with predictions from continuum beam theory. An important implication of our results is a severe reduction of the effective modulus due to buckling, an effect that could be important to interpret experimental results of ultralong amyloid fibrils. Our model represents a powerful tool to mechanically characterize molecular structures on the order of hundreds of nanometers to micrometers on the basis of the underlying atomistic behavior. The work provides insight into structural and mechanical properties of amyloid fibrils and may enable further analysis of larger-scale assemblies such as amyloidogenic bundles or plaques as found in disease states.

Keywords Amyloid · Coarse-grain .

Molecular simulation · Nanomechanics · Materiomics

\section{Introduction}

Amyloid fibrils and plaques are directly related to many neurodegenerative disorders including Alzheimer's disease (AD) [1] and Parkinson's disease [2]. They generate from the conversion of a large number of different proteins from their native functional and soluble state to a highly stable configuration, characterized by a cross-beta geometry and stabilized by a dense network of hydrogen bonds (H-bonds) [3]. This conversion implies the dramatic loss of both solubility and functionality of the proteins. On the other hand, the amyloids, which in a first stage appear in the form of fibrils, show remarkable mechanical properties, including high elasticity, stiffness and resistance [4-6] that render them good candidates as new novel nano-bio-materials. The beta-rich secondary structure of the fibrils correspond to a first level of hierarchical organization that many other materials in nature, including silk, bone and hair [7], feature (Fig. 1). 


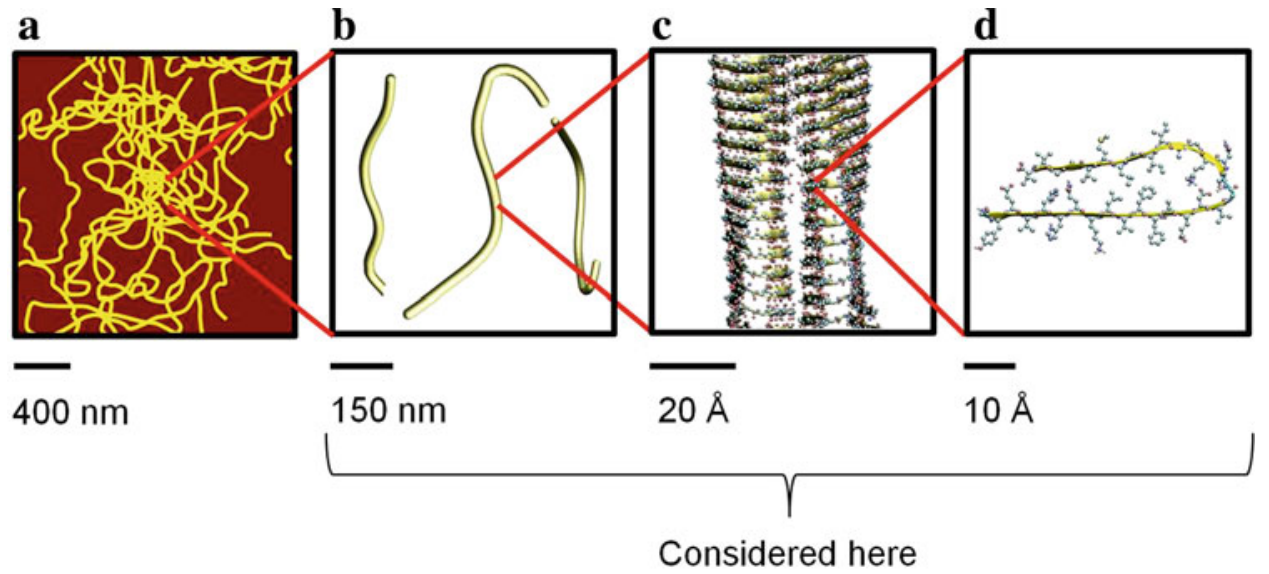

Fig. 1 Multiscale representation of amyloid fibrils, from the nano to the atomistic scale. a Clusters of $\mathrm{A} \beta(1-40)$ amyloid fibrils at the nanoscale. b Representation of individual amyloid fibrils, with lengths of several hundred nanometers. c Atomistic details of the structure of the $\mathrm{A} \beta$ (1-40) amyloid fibril. Each layer is not covalently bonded to its

Recent progress in solid state Nuclear Magnetic Resonance (ssNMR) experiments has provided information on the amyloid core atomistic structure [8], which consists of parallel beta-sheets stacking in register and stabilized solely by the H-bond network. One or more fibrils arrange to form a protofilament [9-11] and the assembly of multiple protofilaments results in morphologies ranging from twisted rope-like structures to flat-tapes with nanometer-scale diameters $[12,13]$. This hierarchical organization (Fig. 1) suggests that the observed properties at the macroscale might directly depend on the interactions and structural details at the atomistic level. In particular, the transfer of the mechanical properties of amyloids through different levels of organization remains unknown and critical to the understanding of the tunable aspects of amyloids, as well as possible candidates for application in nanotechnology. Here we focus on the amyloid fibrils formed by $\mathrm{A} \beta(1-40)$ peptide, which is one of the most abundant species found in $\mathrm{AD}$ brain tissue [14]. These fibrils have been fully investigated both theoretically [15-17] and experimentally [4,18-20], revealing the intriguing mechanical properties (elasticity, sturdiness [4$6,21]$ and self-healing capacity [22-25]) that amyloids show regardless of their chemical structure [4].

Recent results of atomistic simulations that investigated the mechanical properties of amyloid fibrils have revealed the key structural rearrangements (such as a twist-stretch coupling) during loading which are responsible for the high stiffness and strength $[6,21]$. The same investigation at the mesoscale fibrillar level becomes computationally unaffordable given the incredibly high number of atoms and corresponding interactions that should be taken into account. We propose an atomistic informed coarse-grain model that provides an efficient method to simulate and investigate systems neighbors, and interacts solely via the network of H-bonds that connect individual layers. $\mathbf{d}$ Pairs of peptides arranged in a beta-turn structure form individual layers in the amyloid fibril, where the structure of this peptide is stabilized by an internal salt bridge

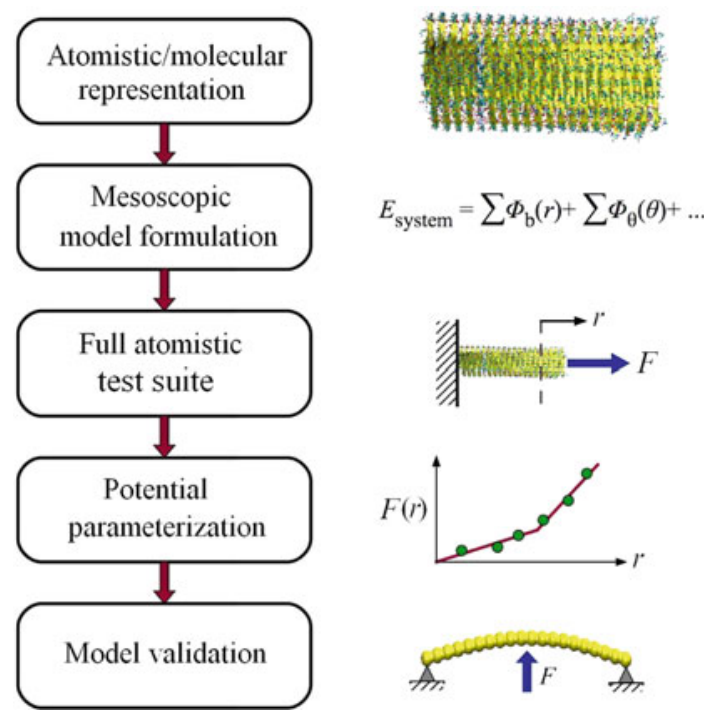

Fig. 2 Schematic of the general steps in the development of the mesoscale model for $\mathrm{A} \beta(1-40)$ amyloid fibrils. Although the atomistic and molecular structure of the fibrils is known, simulation and computational investigations are limited to relatively short fibrils. A mesoscopic formulation is proposed, maintaining elastic behavior for uniaxial strain and bending. A full atomistic "test suite" is applied via MD, characterizing the required mechanical behavior for the mesoscale model, such as axial stiffness and bending rigidity. From the results of the MD simulations, energy equivalence is imposed to parameterize the free variables of the coarse-grain potentials in a "finer-trains-coarser" approach. Finally, to verify and validate the mesoscopic model, simple coarse-grain simulations are undertaken to ensure consistent mechanical behavior and model stability under various load and boundary conditions

with length scales beyond the capacity of the full atomistic simulations, and in particular the characterization of the successive levels of organizations. The general steps of our multiscale approach are schematically represented in Fig. 2. 
A full atomistic "test suite" is applied via molecular dynamics (MD), characterizing the required mechanical behavior for the mesoscale model, such as axial stiffness and bending rigidity. From the results of the MD simulations, energy equivalence is imposed to parameterize the free variables of the coarse-grain potentials in a "finer-trains-coarser" approach. Finally, to verify and validate the mesoscopic model, simple coarse-grain simulations are undertaken to ensure consistent mechanical behavior and model stability under various load and boundary conditions. This tool could reveal very useful information on the perspective of the fabrication of amyloid-based materials with controlled geometries and mechanical properties, which is a promising endeavor [26]. Supplementary to such synthesis efforts, coarse-grain computational approaches can provide an efficient means to investigate potential amyloid fibril structures, indicating possible systems and configurations that warrant experimental and physical realization.

Due to such prevalence, there is a strong motivation to provide an accurate account of the material properties from the bottom up, a key issue in tuning the properties of amyloid fibrils for biomedical and engineering applications. Here, we address this issue and focus on the bottom-up development of a mesoscopic bead-spring model, parameterized to represent individual amyloid fibrils. We then proceed to apply our model to characterize the mechanical properties of fibrils, including the buckling behavior and length dependence, to illustrate the scales unattainable by traditional MD methods. To the best of our knowledge, this is the first mesoscopic model developed to extend the computational analysis of amyloids at larger scales and to higher levels of the materials' organization.

\section{Materials and methods}

\subsection{Full atomistic characterization}

A "fine-trains-coarse" multi-scale approach is implemented to develop a mesoscale model derived solely from full atomistic calculations. A series of full atomistic calculations of mechanical test cases (test suite) is implemented via classical MD to derive a simplified set of parameters to describe the fibril behavior. Relatively simple atomistic simulations are used to isolate a single molecular behavior, mimicking physical material specimen tests. As an analogy, such multiscale model transitions can be compared to the parameterization of atomistic force fields by detailed quantum mechanical methods. It is possible to derive the force constant parameters used in full atomistic models from first principle quantum mechanical calculations [27]. The direct application of quantum mechanical results to large macromolecular systems (such as proteins) is not yet feasible because of the large number of atoms involved. As a logical extension, we analyze the results of full atomistic simulations and implement them in the fitting of mesoscopic coarse-grain potentials. The exact distribution and details of individual amino acid interactions are lost, but the behavior of the molecular structure in its fibril geometry is maintained.

The $\mathrm{A} \beta$ amyloid fibrils consist of layers composed of two $\beta$-units stacking in register along the fiber axis $[18,28]$. Each layer is not covalently bonded to the others, and the overall fibril is stabilized by the dense H-bond network characteristic of the $\beta$ secondary structure. A recent experimentally validated theoretical model provides the structure of fibrils and fibers at the atomistic resolution, and thereby allows the mechanical characterization through computational tools $[6,21]$ with results in good agreement with the experimental findings [4]. A 20 layer fibril is used here as the basis to develop parameters for the coarse grain representation. The choice of a 20 layer fibril is justified by recent theoretical predictions showing that the geometrical, chemical and energetic properties of fibrils of this length can be extended to any size scale [17].

The atomistic test suite used here consists of tensile and compressive loading, to determine uniaxial deformation behavior [6]. Based on this analysis, the results of the full atomistic simulations investigating the mechanical behavior of the $\mathrm{A} \beta(1-40)$ amyloid fibrils [6] are reported in Fig. 3. The mechanical response of a 20 layer amyloid fibril is analyzed under both compressive and tensile loading applying a constant force in the direction of the fibril axis on all the alpha carbon atoms composing the top layer of the fibril, while the bottom of the fibril is fixed. An increase of stiffness is observed in both compression and tension [6]. Although the nonlinear behavior is a result of complex atomistic interactions, it is a trivial simplification to integrate the desired effect into the coarse-grain potential. We can determine Young's modulus either directly from stress-strain results, or indirectly via force-displacement results, depending on the output and sophistication of the full atomistic simulation

$E=\frac{\partial \sigma}{\partial \varepsilon} \approx \frac{\Delta \sigma}{\Delta \varepsilon}=\frac{L_{0}}{A_{\mathrm{c}}} \frac{\Delta F}{\Delta L}$,

where $\sigma$ and $\varepsilon$ are the stress and strain, $\Delta F$ and $\Delta L$ are the force and displacement, and $A_{\mathrm{c}}=b \times h$ and $L_{0}$ are the cross-sectional area (assumed constant, Fig. 4c) and initial length, respectively. The stress-strain behavior of $\mathrm{A} \beta$ (1-40) amyloid fibril under tensile and compressive loading shows an increase of the Young's modulus from 2.34 GPa (for less than $0.168 \%$ strain in compression and $0.266 \%$ strain in tension) to $12.43 \mathrm{GPa}$ for compression and $18.05 \mathrm{GPa}$ for tension [6]. Other full atomistic simulations have been performed to study the mechanical response of these fibrils under bending loading (data not shown). A typical three point bending experiment is performed fixing the alpha carbon 

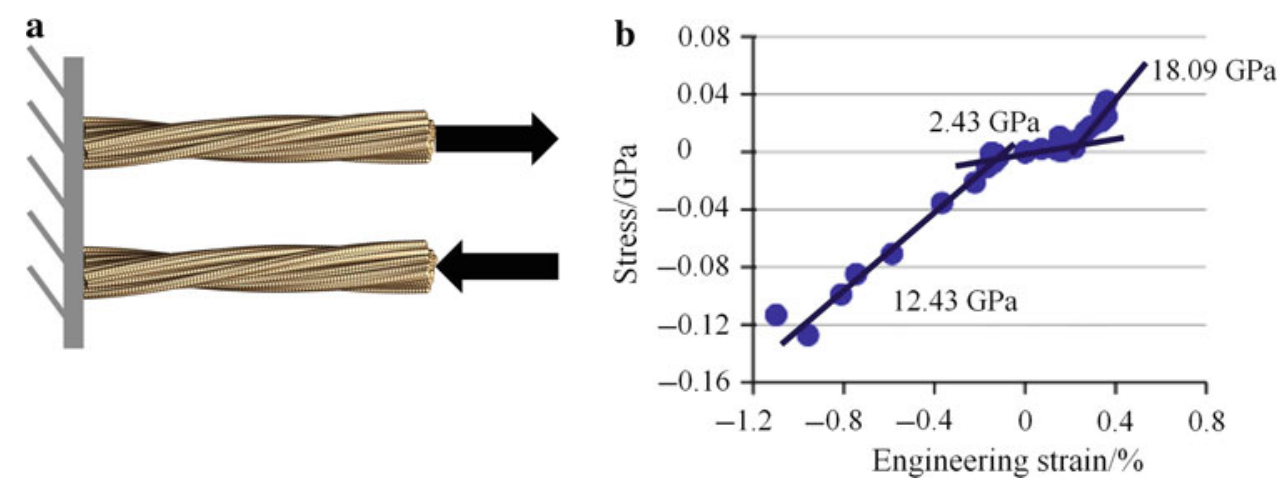

Fig. 3 Mechanical response of a 20 layer $\mathrm{A} \beta$ (1-40) amyloid fibril under compressive and tensile loading at the atomistic scale [6]. a Schematic representation of the computational experiment performed on a $9.1873 \mathrm{~nm}$ long amyloid fibril in tension (top) and compression (bottom). A constant force is applied in both cases on the alpha carbon atoms composing the top layer, while the backbones composing the bottom are fixed. b Mechanical response of the fibril under compressive and tensile loading. An increase of stiffness is observed for strains larger than $0.168 \%$ in compression, and $0.266 \%$ in tension a

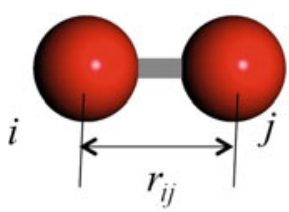

b

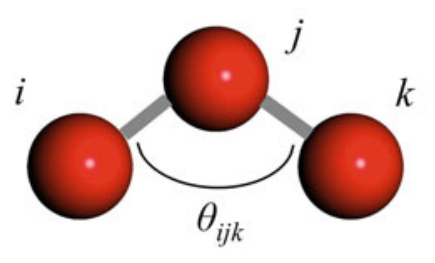

c

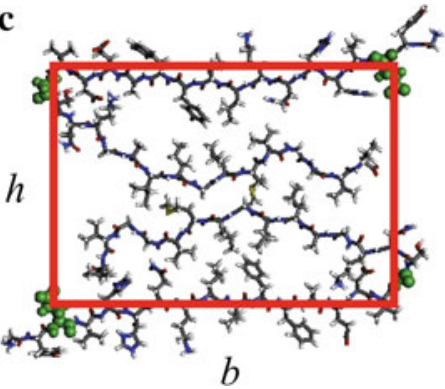

Fig. 4 Formulation of the atomistic informed mesoscale model. a The bond potential contributions. b The angle potential contributions. $\mathbf{c}$ The bending stiffness $E I$, needed for the calculation of the angular potential, is evaluated considering the moment of inertia $I$ of the amyloid fibril cross section represented and approximated as a rectangle

potentially provide an efficient means to investigate the mechanical properties of similar fibrous systems at both meso- and micro-scales, such as fiber materials and nanoscale fabrics.

The fundamental principle underlying the parameterization of a coarse-grain representation is the assertion of energy conservation between full atomistic behavior and coarse-grain potentials. Thus, energy equivalence is imposed between potential energy functions and relevant atomistic results. The basis of the formulation of the energy functions, either through elastic strain energy, deformation energy, adhesion energy, or other techniques, essentially defines the accuracy and behavior of the representative coarse-grain system. The mesocale model is represented by a function of the total energy of the system $\left(E_{\text {system }}\right)$ resulting from two different contributions: $E_{\text {bonds }}$, the energy stored in the chemical bonds due to axial deformation, and $E_{\text {angles }}$, the energy due to bending. It is expressed as

$$
E_{\text {system }}=E_{\text {bonds }}+E_{\text {angles }} \text {. }
$$

The axial and bending contributions to the total energy are calculated by a sum over all pair-wise (distance) and triple (angular) interactions in the system, respectively. We 
proceed to outline the derivation of each potential and the corresponding parameterization.

\subsubsection{Axial stiffness}

The axial potential, $E_{\text {bonds }}$, effectively represents uniaxial deformation (Fig. 4a). At the atomistic scale, there can be many contributions to such a simple mechanical process (such as breaking of hydrogen bonds or solvent friction during molecular stretching). Here, we homogenize such fluctuations to a single mesoscopic behavior, and apply energy equivalence between the coarse-grain potential and the observed strain or deformation energy of the full atomistic system, letting

$E_{\text {bonds }}=\sum_{\text {bonds }} \phi_{\mathrm{b}}(r)$,

and

$\phi_{\mathrm{b}}(r)=U(\varepsilon)$,

where $r$ is the bond distance, $\phi_{\mathrm{b}}(r)$ is again the coarse-grain potential, and $U(\varepsilon)$ is the elastic energy function for the mechanical response. It is an underlying assumption of the model that the response of the full atomistic system is accurately described by the energy function, $U(\varepsilon)$. The choice of the strain or deformation energy function is dependent on the system to be coarse-grained: we apply a bilinear elastic model for uniaxial stretching, due to the form of the full atomistic results [6]. To equate with $\phi_{\mathrm{b}}(r)$, we must formulate $U$ as a function of $r$. For axial stretching, this requires the formulation of strain as a function of bond length, $\varepsilon(r)$. Assuming elastic-isotropic behavior, we can define strain energy as

$$
\begin{aligned}
U(\varepsilon) & =\frac{1}{2} \int_{\bar{V}}(\sigma \varepsilon) \mathrm{d} \bar{V}=\frac{1}{2} \frac{A_{\mathrm{c}} E \Delta r^{2}}{r_{0}} \\
& =U(\Delta r)=U\left(r-r_{0}\right)=U(r),
\end{aligned}
$$

with $r_{0}$ being the bond equilibrium distance. For the integration over the volume, $\bar{V}$, we assume a constant cross-section, $A_{\mathrm{c}}$, such that $\bar{V}=A_{\mathrm{c}} r_{0}$, define strain, $\varepsilon=$ $\Delta r / r_{0}$, and stress, $\sigma=E \varepsilon$. We note that we utilized the full atomistic simulations to determine Young's modulus specifically to allow this formulation of strain energy in our parameterization. Caution must be taken not to overextend the significance of the atomistic to continuum equivalence. Here, we only use Young's modulus to characterize the work required to stretch our atomistic model and thus train the coarse-grain potential. It is not implied that amyloidogenic fibrils can be suitably modeled by elastic formulations. Indeed, unlike the Young's modulus of elastic isotropic materials, the modulus determined by atomistic simulation can differ depending on system properties, atomistic force field, boundary conditions, and loading rates [33].
The total bond energy of the coarse-grain system is given by the sum over all bonded interactions as in Eq. (3a). For axial stretching a simple harmonic spring is used to determine the energy between all bonded pairs of particles in the system, given by

$\phi_{\mathrm{b}}(r)=\frac{1}{2} k_{\mathrm{b}}\left(r-r_{0}\right)^{2}$,

with $k_{\mathrm{b}}$ as the spring constant related to the distance $r$. We assume each linear regime can be approximated using the equivalent elastic strain energy and utilize the determined Young's modulus from full atomistic simulations to allow the formulation of strain energy and an equivalent $k_{\mathrm{b}}$. The contributions $\phi_{\mathrm{b}}$ to the bonding energy can be written as follows

$$
\phi_{\mathrm{b}}(r)=\frac{1}{2} \begin{cases}k_{\mathrm{b}}^{\mathrm{s}}\left(r_{\text {stiff }}^{\mathrm{c}}-r_{0}\right)^{2}+k_{\mathrm{b}}^{\mathrm{c}}\left(r-r_{\text {stiff }}^{\mathrm{c}}\right)^{2}, & r \leq r_{\text {stiff }}^{\mathrm{c}}, \\ k_{\mathrm{b}}^{\mathrm{s}}\left(r-r_{0}\right)^{2}, & r_{\text {stiff }}^{\mathrm{c}}<r<r_{\text {stiff }}^{\mathrm{t}}, \\ k_{\mathrm{b}}^{\mathrm{s}}\left(r_{\text {stiff }}^{\mathrm{t}}-r_{0}\right)^{2}+k_{\mathrm{b}}^{\mathrm{t}}\left(r-r_{\text {stiff }}^{\mathrm{t}}\right)^{2}, & r \geq r_{\text {stiff }}^{\mathrm{t}} .\end{cases}
$$

This function allows us to model the changes in the elastic properties during the deformation. The parameter $k_{\mathrm{b}}^{i}$ represents the spring constant relative to the strain range $i$ (the superscribts meaning $\mathrm{c}=$ compression, $\mathrm{t}=$ tension, $\mathrm{s}=$ small deformations). For equivalent energy and consistent mechanical behavior, we let $\phi_{\mathrm{b}}(r)=U(r)$ and find

$k_{\mathrm{b}}^{i}=\frac{A_{\mathrm{c}} E^{i}}{r_{0}}$,

where $E^{i}$ indicates the effective Young's modulus to account for the nonlinear regimes. Using the Young's moduli $\left(E^{i}\right)$ listed above, the spring constants are $k_{\mathrm{b}}^{\mathrm{c}}=138.216 \mathrm{~N} / \mathrm{m}$, $k_{\mathrm{b}}^{\mathrm{s}}=26.002 \mathrm{~N} / \mathrm{m}$ and $k_{\mathrm{b}}^{\mathrm{t}}=200.719 \mathrm{~N} / \mathrm{m}$. For the model, a bead comprises three layers and it is assumed that each has a constant cross section area $A_{\mathrm{c}}=14.1432 \mathrm{~nm}$ (Fig. 4c) and is set at a distance $r_{0}=1.2717 \mathrm{~nm}$ from its nearest neighbors [6]. From the full atomistic data, values of the distances corresponding to an increase of stiffness in both tension $\left(r_{\text {stiff }}^{\mathrm{t}}\right)$ and compression $\left(r_{\text {stiff }}^{\mathrm{c}}\right)$ are calculated by considering a stiffness change at $0.266 \%$ strain in tension and $0.168 \%$ strain in compression. The critical distances are $r_{\text {stiff }}^{\mathrm{t}}=1.2750 \mathrm{~nm}$ and $r_{\text {stiff }}^{\mathrm{c}}=1.2695 \mathrm{~nm}$.

\subsubsection{Bending stiffness}

For the angle potential, $E_{\text {angles, }}$, the bending stiffness and force-displacement behavior are required (Fig. 4b). The potential is intended to reflect the bending rigidity (or bending stiffness) of the amyloid fibril. Here, we approximate the bending rigidity of an amyloid fibril by formulating the behavior as equivalent to Euler-Bernoulli beam theory, commonly applied to biological filaments and nanostructures. 
Again, such an assumption is not intended to imply the fibril behaviors identically to classical beam deflection, but rather a means to approximate the elastic energy of bending deformation. We proceed to apply energy equivalence between the coarse-grain potential and the idealized bending strain energy of the full atomistic system, letting

$E_{\text {angles }}=\sum_{\text {angles }} \phi_{\mathrm{t}}(\theta)$

and

$\phi_{\mathrm{t}}(\theta)=U(d)$

where $d$ represents the bending displacement and $\theta$ the bending angle between three particles relative to the equilibrium angle, $\theta_{0}=180^{\circ}$. From the results, we can determine the bending stiffness of the molecule, which we label EI. The bending energy is given by a sum over all triples in the system as Eq. (8a). For bending a rotational harmonic spring potential is used to determine the energy between all triples of particles in the system

$\phi_{\mathrm{t}}(\theta)=\frac{1}{2} k_{\mathrm{t}}\left(\theta-\theta_{0}\right)^{2}$,

with $k_{\mathrm{t}}$ as the spring constant related to the bending angle $\theta$. Again, using the equivalent elastic energy, we utilize the bending stiffness, $E I$, from full atomistic results to allow the formulation of elastic energy and an equivalent $k_{\mathrm{t}}$.

It has been shown that the shear contribution can affect the bending behavior of such beta-sheet filament structures [34], requiring Timoshenko-type beam-deflection theories [35] to accurately capture the force-displacement behavior. However, the intent of the mesocopic formulation applied here is specifically to extend the length-scale of the modeled amyloid fibrils in which the classical beam theory is deemed suitable.

Computationally, the angle potential computes restoring forces to be applied to each particle involved in the angle triple interaction. The restoring forces act normal to the direction of the bond, essentially reproducing three-point bending conditions. From Euler-Bernoulli theory

$d=\frac{P L^{3}}{48 E I}$,

with $P$ corresponding to the applied bending load on the fibril. Using the equivalent elastic energy [36], the work due to bending can be expressed as

$U(d)=\frac{48 E I}{\left(2 r_{0}\right)^{3}} d^{2}$.

For small deformation, $\theta-\theta_{0} \approx 2 d / r_{0}$, and letting $\phi_{\theta}(d)=$ $U(d)$ $k t=\frac{3 E I}{r_{0}}$,

and it results to be $6.08 \times 10^{-17} \mathrm{~J}$, using a moment of inertia of $I=110,070.81 \times 10^{-40} \mathrm{~m}^{4}[6]$ and a Young's modulus $(E)$ and equilibrium spacing $\left(r_{0}\right)$ previously determined. It is noted that based on the resulting bending stiffness, the persistence length of the amyloid fibrils is estimated to be larger than $6 \mu \mathrm{m}[6]$.

\subsubsection{Bead properties}

Assuming a homogenous distribution of mass in the molecular model, the corresponding mass can be calculated by the summation of the masses of all the atoms composing the three included layers $(30,940.98 \mathrm{~g} / \mathrm{mol})$. The "fine-trains-coarse" approach eliminates the reliance on empirical parameter tuning. From atomistic simulations using scales of femtoseconds and Angstroms, a set of mesoscopic parameters was derived as outlined above. The resulting mesoscale model enables modeling of the dynamics of systems of ultra long amyloid fibrils over time scales approaching microseconds, facilitating a bridge between atomistic theory and simulation of actual physical experiments. The mesoscopic model for fibrils can be defined by four parameters: $k_{\mathrm{b}}^{i}, r_{0}, k_{\mathrm{t}}$ and $\theta_{0}$. The results from the above atomistic simulations are used to determine the parameters via equilibrium conditions $\left(r_{0}, \theta_{0}\right)$ and energy conservation $\left(k_{\mathrm{b}}^{i}, k_{\mathrm{t}}\right)$. Because of the use of a multi-scale approach, the atomistic behavior and intramolecular interactions are maintained, thereby providing a necessary intermediate step reconciling the gap between atomistic and continuum theory.

The mesoscale model is implemented in the massively parallelized code LAMMPS (http://lammps.sandia.gov/) and the results presented here have been carried out on a single CPU Linux workstation. The force field is modified introducing multi-linear harmonic bond potentials as previously described. We note that since the basis of the model is atomistic simulation, the multi-linear spring constants implicitly take into account the effects of solvent and molecular friction, while additional parameters for viscosity and other effects are not considered here. The LAMMPS code has been extended to take into account the variation of the elastic properties with the deformation as discussed. The parameters calculated as described above are summarized in Table 1.

\section{Results and discussion}

\subsection{Model validation}

Once the coarse-grain potentials are developed, validation of the coarse-grain model is a necessary step to 
Table 1 Summary of mesoscale model parameters

\begin{tabular}{ll}
\hline Parameter & Value \\
\hline Tensile stiffness, $k_{\mathrm{b}}^{i}\left(\mathrm{~N} \mathrm{~m}^{-1}\right)$ & 26.002 \\
& 138.216 (Compression) \\
& 200.719 (Tension) \\
Bending stiffness, $k_{\mathrm{t}}(\mathrm{J})$ & $6.08 \times 10^{-17}$ \\
Equilibrium bead distance, $r_{0}(\mathrm{~nm})$ & 1.2717 \\
Critical bead distance, $r_{\text {stiff }}^{\mathrm{t}}(\mathrm{nm})$ & 1.2750 \\
Critical bead distance, $r_{\text {stiff }}^{\mathrm{c}}(\mathrm{nm})$ & 1.2695 \\
Equilibrium angle, $\theta_{0}\left(^{\circ}\right)$ & 180 \\
\hline
\end{tabular}

assure an accurate representation. The primary basis for validation is through a direct comparison to full atomistic simulations. Such an approach may seem counterintuitive, redundant and/or self-serving, as the coarse-grain potentials were developed from such simulation results. However, the "test suites" applied were intended to result in a single behavior/response for each coarse-grain potential. The full coarse-grain representation can possibly involve the interaction and/or coupling of individual potentials. The combination of two behaviors (bending and stretching for example) must be explored to justify the coarse-grain model. Relatively simple validation simulations can be designed, combing multiple system behaviors, and a one-to-one comparison made between full atomistic and coarse-grain results.

Coarse grained amyloid fibrils, with length of about $24 \mathrm{~nm}$ are built and mechanical tests are performed to validate our mesoscale model. In particular, both tension and compression loading are carried out fixing one of the tails of the fibrils (as represented in Fig. 5a) and imposing a velocity of $1 \mathrm{~m} / \mathrm{s}$ to the bead constituting the other tail. The plot reported in Fig. 5b illustrates the mechanical response in both compression and tension, showing a very good agreement with the atomistic results (Fig. 3) for both the profile of the curve and the found Young's moduli in the three regimes (small deformation, compression and tension large deformations). Due to limitations of full atomistic simulations, such validation is restricted to component-level behavior in which the coarse -grain model is specifically developed to circumvent. If available, secondary support and model validation is found in experimental data. Experimental techniques such as nanoindentation or atomic/chemical force microscopy (AFM/CFM) can directly probe materials at the mesoscale and determine system level characteristics and mechanical properties. The development of coarse-grain models is intended to elucidate such system-level behavior and thus correlation with experimental results is essential. Reciprocally, an accurate coarse-grain model can serve to validate and support experimental results. The full atomistic simulations and the subsequent studies at the mesoscale level generates elastic constants, which are in the same range of the experimental data, which reported a Young's modulus for the amyloids in the range 10-20 GPa [4].

The mesoscale settings take into account the elasticity and the stiffness driving the general behavior of amyloids and can be used to study systems with variable complexity, order and size. A developed coarse-grain model can only reflect the behavior included in their governing potentials and associated parameters, and consequently, the source of such parameters typically determines the accuracy and utility of the coarse-grain model.

\subsection{Mechanical properties under compressive loading}

We now use the model to investigate the dependence of the mechanical behavior under compressive loading, as a function of the length of the amyloid fibril. In Fig. 6, compressive stress-strain curves of single amyloid fibrils with lengths of $38.2 \mathrm{~nm}$ (Fig. 6a) and $62.3 \mathrm{~nm}$ (Fig. 6b) are depicted. In both cases, the occurrence of buckling is accompanied by a sudden change of the stress-strain profile following an initial linear elastic regime. As shown in Fig. 6, longer fibrils experience larger fluctuations during compression. The buckling of shorter fibrils requires a higher critical stress $\sigma_{\text {buckl }}$, while
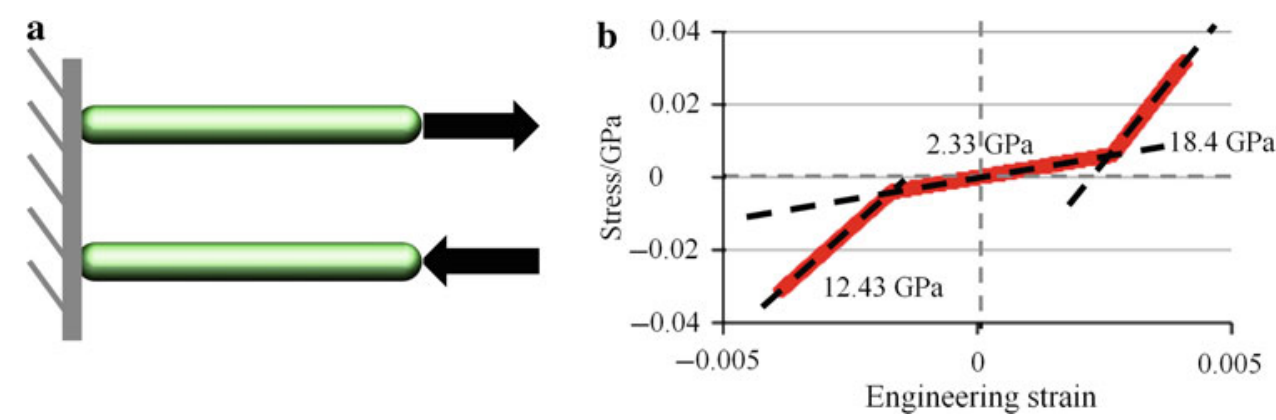

Fig. 5 Validation of the mesoscale model. a Loading geometry of a single, approximately $24 \mathrm{~nm}$ long fibril in tension and compression. One of the two tails of the fibril is fixed, while a constant velocity of $1 \mathrm{~m} / \mathrm{s}$ is applied in tension (top) and compression (bottom) on the other.

b Mechanical response under compressive and tensile loading. As in the atomistic simulations, there is an increase of stiffness for strain larger than $0.266 \%$ in tension and $0.168 \%$ in compression, and the values of the elastic moduli are well reproduced in the mesoscale model 


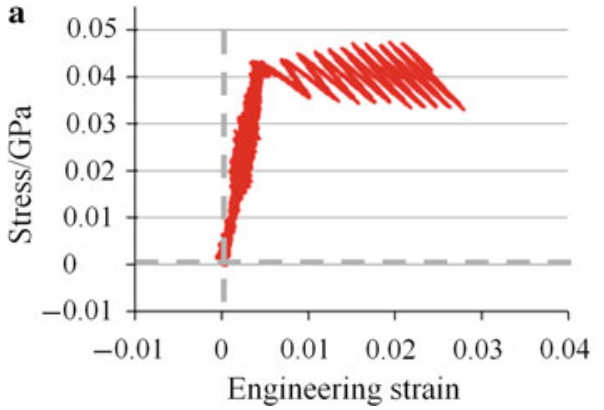

Fig. 6 Mechanical response of amyloid fibrils under compression (loading as shown in the lower part of Fig. 5a). a, b Stress-strain plots of $38.2 \mathrm{~nm}$ and a $62.3 \mathrm{~nm}$ long amyloid fibrils, respectively. Longer fib-

a

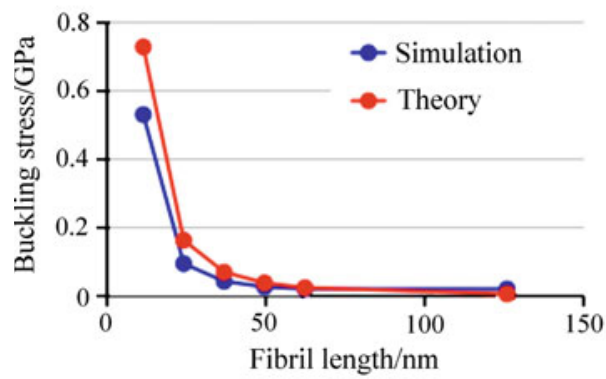

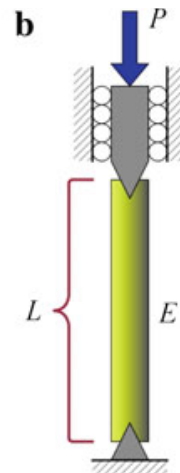

Fig. 7 Mechanical response of amyloid fibrils under compressive loading. a Comparison between beam theory predictions and simulation results at the mesoscale of the buckling stress as a function of the fibril length. b Application of mechanical boundary conditions. In the computational experiments, a constant displacement rate is applied to one of the fibril tails, while the other is pinned but free to rotate

it occurs at lower values in longer fibrils. Thus, the buckling process implies an apparent softening of the fibrils due to the fluctuations occurring during loading.

Figure 7 shows a systematic analysis of the buckling stress measured for amyloid fibrils with lengths in the range of $10-125 \mathrm{~nm}$. A compressive constant velocity of $1 \mathrm{~m} / \mathrm{s}$ is applied to one of the two tails, previously shifted by an imposed eccentricity of $1 \%$ of the fibril length, in the direction perpendicular to the fibril, while the other tail is fixed (boundary conditions shown in Fig. 7b). The simulations are performed until the occurrence of buckling is confirmed. The same analysis on longer fibrils is limited by the onset of large fluctuations of the structure during compression, which makes the analysis of the corresponding stress-strain plot increasingly difficult.

On the basis of beam theory, the critical buckling stress can be defined as

$\sigma_{\text {buckl }}=\frac{\pi^{2} E I}{A_{\mathrm{c}}(\alpha L)^{2}}$

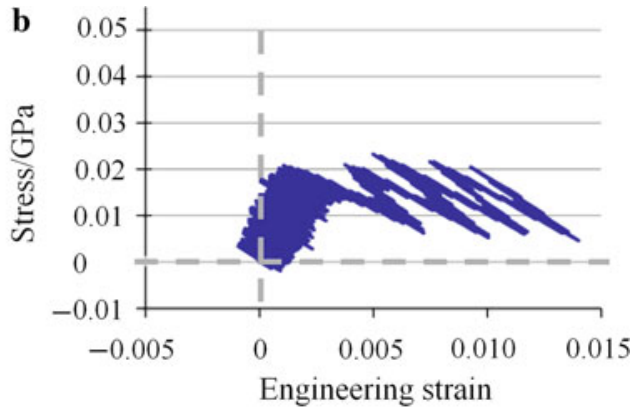

rils present larger fluctuations and buckling at lower stress. The same stress scale is used for $\mathbf{a}$ and $\mathbf{b}$ to highlight the difference amount of fluctuations that occur at distinct fibril lengths

where the parameter $\alpha$ denotes the effective length scaling factor based on the boundary conditions of the buckling mode. The moment of inertia $I$, and cross-sectional area $A_{\mathrm{c}}$ are estimated from the atomistic model (Fig. 4c) described in [6]. The data reported in Fig. 7a show a comparison between the theoretical predictions and the simulation results of the critical stress, revealing a reasonable agreement between the two sets of data and confirming the validity of the presented mesoscale model. The extension of the model to describe longer fibrils could be implemented in future works. Given the computational cost associated with our model it could directly simulate amyloid fibrils close to their persistence length (in the range 6-48 $\mu \mathrm{m}$ for amyloid fibrils [6]). This could be interesting to study the interplay of different deformation and failure mechanisms.

\section{Conclusion}

The proposed mesoscale model allows the investigation of large systems that otherwise could not be studied using full atomistic simulations. Despite the level of approximation and the loss of the atomistic details and of the molecular interactions, the mechanical behavior can still be described well at larger scales. The validation of the mesoscale model and the agreement of the mechanical response of coarse grained systems with the results of full atomistic simulations not only demonstrate the correctness of our approach, but also show how the transfer of the information from the atomistic to the mesoscopic level can be used to investigate systems reaching lengths of several hundreds of nanometers.

The buckling analysis presented in this paper can be used to complete a systematic mechanical characterization of the amyloid single fibril with varying length. The good agreement with the beam theory results (as shown in Fig. 7) shows that the introduced approximations in the coarse-grained model do not affect the validity of the analysis, despite the extreme simplicity of the model, which implies only two contributions (axial stretching and bending stiffness). A further 
development of the model would extend to the adhesion energy involved in the interactions of different fibrils or of different parts of the same fibril and would allow the analysis of amyloid bundles, protofilaments and plaques. This analysis is left to future work. Moreover, the observation of the size dependence of the fibrils mechanical response under compressive loading might suggest new experimental studies and shed light on the mechanical properties of long fibrils, which must be carefully investigated noting the described mechanisms such as buckling.

Specifically, our model provides insight into the structural and mechanical properties of amyloid fibrils approaching the length of a few hundred of nanometers and might therefore enable further analysis of large-scale assemblies, such as amyloids bundles or plaques (for a visual representation of these scales, see Fig. 1). Despite the simplicity of the formulation and the high level of approximation, this approach holds the potential to describe the mechanical behavior of the fibrils at ultra-large scales and thus opens a novel perspective of a systematic theoretical study of amyloid aggregation for both advanced applications in nanotechnology and the improvement of the understanding of the interactions between large amyloid plaques and cells composing soft tissue (e.g. neuron cells). Future work could include studies of the effect of compression velocity, the influence of the twisted geometry of amyloid fibrils, the role of local defects as well as the effect of thermal fluctuation on the compressive properties of amyloid fibrils.

\section{References}

1. Chiti, F., Dobson, C.M.: Protein misfolding, functional amyloid, and human disease. Annu. Rev. Biochem. 75, 333-366 (2006)

2. Braak, H., Braak, E.: Cognitive impairment in parkinsons-diseaseamyloid plaques, neurofibrillary tangles, and neuropil threads in the cerebral-cortex. J. Neural Transmission Parkinsons Dis. Dement. Sect. 2(1), 45-57 (1990)

3. Goldschmidt, L., Teng, P., Riek, R., et al.: The amylome, all proteins capable of forming amyloid-like fibrils. Proc. Natl. Acad. Sci. USA 107(8), 3487-3492 (2010)

4. Knowles, T.P., Fitzpatrick, A.W., Meehan, S., et al.: Role of intermolecular forces in defining properties of protein nanofibrils. Science 318(5858), 1900-1902 (2007)

5. Smith, J.F., Knowles, T.P., Dobson, C.M., et al.: Characterization of the nanoscale properties of individual amyloid fibrils. Proc. Natl. Acad. Sci. USA 43(103), 15806-15811 (2006)

6. Paparcone, R., Keten, S., Buehler, M.J.: Atomistic simulation of nanomechanical properties of Alzheimer's A $\beta$ (1-40) amyloid fibrils under compressive and tensile loading. J. Biomech. 43(6), 1196-1201 (2010)

7. Alberts, B., Johnson, A., Lewis, J., et al.: Molecular Biology of the Cell. Garland Science, New York (1994)

8. Nelson, R., Sawaya, M.R., Balbirnie, M., et al.: Structure of the cross-beta spine of amyloid-like fibrils. Nature 435, 773-778 (2005)
9. Jimenez, J.L., Nettleton, E.J., Bouchard, M., et al.: The protofilament structure of insulin amyloid fibrils. Proc. Natl. Acad. Sci. USA 99, 9196-9201 (2002)

10. Jimenez, J.L., Guijarro, J.I., Orlova, E., et al.: Cryo-electron microscopy structure of an SH3 amyloid fibril and model of the molecular packing. EMBO J. 18, 815-821 (1999)

11. Serpell, L.C., Sunde, M., Benson, M.D., et al.: The protofilament substructure of amyloid fibrils. J. Mol. Biol. 300, 1033-1039 (2000)

12. Knowles, T.P.J., Smith, J.F., Craig, A., et al.: Spatial persistence of angular correlations in amyloid fibrils. Phys. Rev. Lett. 96, 238301 (2006)

13. Aggeli, A., Nyrkova, I.N., Bell, M., et al.: Hierarchical self-assembly of chiral rod-like molecules as a model for peptide $\beta$-sheet tapes, ribbons, fibrils, and fibers. Proc. Natl. Acad. Sci. USA 98, 11857-11862 (2001)

14. Finder, V.H., Glockshuber, R.: Amyloid-beta aggregation. Neurodegener Dis. 4(1), 13-27 (2007)

15. Periole, X., Rampioni, A., Vendruscolo, M., et al.: Factors that affect the degree of twist in beta-sheet structures: a molecular dynamics simulation study of a cross-beta filament of the GNNQQNY peptide. J. Phys. Chem. B 113, 1728-1737 (2009)

16. Buchete, N.V., Tycko, R., Hummer, G.: Molecular dynamics simulations of Alzheimer's beta-amyloid protofilaments. J. Mol. Biol. 353, 804-821 (2005)

17. Paparcone, R., Buehler, M.J.: Microscale structural model of Alzheimer Abeta (1-40) amyloid fibril. Appl. Phys. Lett. 94, 243904 (2009)

18. Petkova, A.T., Ishii, Y., Balbach, J.J., et al.: A structural model for Alzheimer's beta-amyloid fibril based on experimental constraints from solid state NMR. Proc. Natl. Acad. Sci. USA 99(26), 1674216747 (2002)

19. Paravastu, A., Leapman, R.D., Yau, W.M., et al.: Molecular structural basis for polymorphism in Alzheimer's Beta-amyloid fibrils. Proc. Natl. Acad. Sci. USA 105(47), 18349-18354 (2008)

20. Tycko, R.: Insights into the amyloid folding problem from solid-state NMR. Biochemistry 42(11), 3151-3159 (2003)

21. Xu, Z., Paparcone, R., Buehler, M.J.: Alzheimer's A $\beta$ (1-40) amyloid fibrils feature size dependent mechanical properties. Biophys. J. 98, 2053-2062 (2010)

22. Gelain, F., Bottai, D., Vescovi, A., et al.: Designer self-assembling peptide nanofiber scaffolds for adult mouse neural stem cell 3-Dimensional cultures. PLoS ONE 1, e119 (2006)

23. Scheibel, T., Parthasarathy, R., Sawicki, G., et al.: Conducting nanowires built by controlled self-assembly of amyloid fibrs and selective metal deposition. Proc. Natl. Acad. Sci. USA 100(8), 4527-4532 (2003)

24. Zhang, S.: Fabrication of novel biomaterials through molecular self-assembly. Nat. Biotechnol. 21, 1171-1178 (2003)

25. Zhang, S., Holmes, T., Lockshin, C., et al.: Spontaneous assembly of a self-complementary oligopeptide to form a stable macroscopic. Proc. Natl. Acad. Sci. USA 90(8), 3334-3338 (1993)

26. Knowles, T.P., Oppenheim, T.W., Buell, A.K., et al.: Nanostructured films from hierarchical self-assembly of amyloidogenic proteins. Nat. Nanotechnol. 5, 204-207 (2010)

27. Car, R., Parrinello, M.: Unified approach for molecular dynamics and density-functionaly theory. Phys. Rev. Lett. 55(22), 2471-2474 (1985)

28. Ma, B., Nussinov, R.: Stabilities and conformations of Alzheimer's beta-amyloid peptide oligomers (Abeta 16-22, Abeta 16-35, and Abeta 10-35), Sequence effects. Proc. Natl. Acad. Sci. USA 99, 14126-14131 (2002)

29. Buehler, M.J., Duin, A.C.T.v., Goddard, W.A.: Multi-paradigm modeling of dynamical crack propagation in silicon using the ReaxFF reactive force field. Phys. Rev. Lett. 96(9), 095505 (2006) 
30. Cranford, S., Buehler, M.J.: Mechanomutable carbon nanotube arrays. Int. J. Mater. Struct. Integrity 3(2/3), 161-178 (2009)

31. Cranford, S., Yao, H., Ortiz, C., et al.: A single degree of freedom "lollipop" model for carbon nanotube bundle formation. J. Mech. Phys. Solids 58(3), 409-427 (2009)

32. Cranford, S., Sen, D., Buehler, M.J.: Meso-origami: folding multilayer graphene sheets. Appl. Phys. Lett. 95, 122131 (2009)

33. Ackbarow, T., Chen, X., Keten, S.: Hierarchies, multiple energy barriers, and robustness govern the fracture mechanics of $\{\alpha\}$-helical and $\beta$-sheet protein domains. Proc. Natl. Acad. Sci. 104, 16410-16415 (2007)
34. Keten, S., Xu, Z., Ihle, B., et al.: Nanoconfinement controls stiffness, strength and mechanical toughness of beta-sheet crystals in silk. Nat. Mater. 9, 359-367 (2010)

35. Wang, C.M.: Timoshenko beam-bending solutions in terms of euler-bernoulli solutions. J. Eng. Mech. Asce 121(6), 763-765 (1995)

36. Timoshenko, S., Maccullough, G.H.: Elements of Strength of Materials. 2nd edn. D. Van Nostrand Company, New York (1940) 